\title{
Initial 3-Weeks' Apixaban Versus Dual-Antiplatelet Therapy (Clopidogrel and Aspirin) Followed by Clopidogrel Alone in High- Risk Patients with Acute Non-Disabling Cerebrovascular Events (ADANCE): Study Protocol for a Randomized Controlled Trial
}

\author{
Fang Yang $\cdot$ Hui Lei $\cdot$ Wenrui Jiang $\cdot$ \\ Wen Jiang $\cdot$ Junliang Han $\cdot$ Gang Zhao
}

Published online: 9 September 2014

(c) The Author(s) 2014. This article is published with open access at Springerlink.com

\begin{abstract}
Background Nondisabling cerebrovascular events represent the largest group of cerebrovascular disease with a high risk of recurrent stroke. A recent trial demonstrated that dual-antiplatelet therapy (clopidogrel and aspirin), compared with aspirin monotherapy, reduced the risk of recurrent stroke and was not associated with increased risk of hemorrhagic events. Apixaban, a new oral anticoagulant, has been proven to be as safe and effective as traditional anticoagulants while carrying significantly less risk of intracranial hemorrhage. Patients with transient ischemic attack (TIA)/minor stroke might benefit from apixaban
\end{abstract}

F. Yang, H. Lei, and W. Jiang contributed equally.

Trial registration: ClinicalTrials.gov identifier NCT01924325.

F. Yang $\cdot$ W. Jiang $\cdot$ J. Han $\cdot$ G. Zhao $(\bowtie)$

Department of Neurology, Xijing Hospital, No. 15 West Changle

Road, Xi' an 710032, China

e-mail: zhaogang@fmmu.edu.cn

F. Yang

e-mail: fyangx@fmmu.edu.cn

W. Jiang

e-mail: jiangwen@fmmu.edu.cn

J. Han

e-mail: hanjunliang@gmail.com

H. Lei

Department of Neurology, Xi' an Center Hospital, No. 185 North Street, Xi' an 710003, China

e-mail: 29652220@qq.com

W. Jiang

Department of Cardiology, Tangdu Hospital, No. 1 Xinsi Road,

Xi' an 710038, China

e-mail: jiangwr@fmmu.edu.cn treatment; therefore, an adequately powered randomized study is needed.

Methods and Results The ADANCE [Apixaban Versus Dual-antiplatelet Therapy (Clopidogrel and Aspirin) in Acute Non-disabling Cerebrovascular Events] study is a randomized, double-blind clinical trial with a target enrollment of 5,500 patients. A 21-day regimen of apixaban or of clopidogrel with aspirin followed by clopidogrel on days 22 through 90 will be administered to randomized participants with acute TIA or minor ischemic stroke. The primary efficacy endpoint is the percentage of patients with any new stroke (ischemic or hemorrhage), including fatal stroke, at day 21. Study visits will be performed on the day of randomization, and at days 7, 22, and 90 .

Discussion The novel oral anticoagulant apixaban has been widely used with fewer adverse effects than traditional anticoagulants. We designed the ADANCE trial to observe the effects of apixaban on recurrent stroke after TIA or minor stroke. The results should better guide the selection of anticoagulant or dual-antiplatelet therapy for patients with acute TIA or minor ischemic stroke.

\section{Introduction}

Stroke, the leading cause of acquired disability, affects 15 million people worldwide every year [1]. Transient ischemic attack (TIA) and acute minor ischemic stroke, defined as a National Institutes of Health Stroke Scale (NIHSS) score $\leq 3$, represent the largest group of cerebrovascular disease. About $30-40 \%$ of patients with ischemic stroke have had a prior TIA or minor stroke [2]. A previous study suggests that the 90 -day risk of a subsequent stroke is $10.5 \%$ after TIA and that almost half of these strokes could occur within the first 2 days [3]. After a 
minor stroke, the risk of recurrence is as high as $18.5 \%$ at day 90 [4].

Aspirin, the gold standard of medical care for antiplatelet therapy, has been used most extensively for ischemic cerebrovascular diseases. Compared to intravenous tissue plasminogen activator (t-PA), which has a narrow therapeutic window (up to $4.5 \mathrm{~h}$ after symptom onset) [5], and traditional anticoagulants with a higher risk of bleeding events, aspirin has a modest effect on acute ischemic stroke. Compared to placebo, aspirin administration shows a slight increase in the risk of cerebral hemorrhage. The recently published CHANCE (Clopidogrel in High-risk Patients With Acute Non-disabling Cerebrovascular Events) study [6] reported superior results with dual-antiplatelet therapy (DAPT), i.e., superiority of clopidogrel-aspirin over aspirin monotherapy. DAPT was not associated with increased hemorrhage events. The results shed light on the benefits of exploiting more effective medical therapy for patients suffering non-disabling cerebrovascular diseases.

Traditional anticoagulants have been used to lower the risk of early recurrent stroke; however, the benefit is negated by an increasing rate of bleeding complications [7, 8]. Recently, the novel oral anticoagulants have received extensive attention because of the following advantages [9]: (1) they directly target the coagulation cascade with rapid onset/offset of action; (2) fewer adverse effects (especially lower rates of major hemorrhage); (3) lower risk for drug-drug interactions; and (4) a more predictable response. Apixaban, a direct factor Xa inhibitor, as one of these novel oral anticoagulants, has been approved for preventing venous thromboembolism after elective hip or knee replacement in Europe [10] and for reducing the risk of stroke and dangerous blood clots (systemic embolism) in patients with atrial fibrillation (AF) by the US Food and Drug Administration [11]. The ARISTOTLE (Apixaban for Reduction in Stroke and Other Thromboembolic Events in Atrial Fibrillation) trial [12] found that apixaban could reduce the risks of systemic embolism, bleeding events, and all-cause mortality, with better tolerability in patients with AF, compared with warfarin treatment. The AVERROES (Apixaban Versus Acetylsalicylic Acid to Prevent Stroke in Atrial Fibrillation Patients Who Have Failed or Are Unsuitable for Vitamin K Antagonist Treatment) trial [13] confirmed that apixaban was associated with more effective reduction of stroke and a similar risk of major bleeding compared with aspirin in patients with AF. Hence, we speculated that apixaban might show better effect on patients with TIA or acute ischemic stroke.

To date, the effect of apixaban versus DAPT (clopidogrel and aspirin) on acute non-disabling cerebrovascular disease is still unconfirmed. In the ADANCE study, we will enroll patients with TIA and minor ischemic stroke who are showing particularly high risk for recurrent ischemia, low risk for hemorrhage, and relatively stable systematic conditions after cerebrovascular event onset. The study was designed to estimate whether apixaban administration is superior to DAPT, in order to decrease recurrent stroke in Chinese patients after TIA and acute minor stroke.

\section{Study Objective}

An initial 3 weeks of apixaban versus DAPT therapy (clopidogrel and aspirin) followed by clopidogrel alone in high-risk patients with acute non-disabling cerebrovascular event (ClinicalTrials.gov identifier NCT01924325) is a randomized, double-blind, multicenter, controlled clinical trial enrolling 5,500 Chinese patients with acute TIA or minor stroke. The primary objective of the trial is to determine whether apixaban is safe and could reduce the risk of any stroke (both ischemic and hemorrhagic) when initiated within $24 \mathrm{~h}$ of symptom onset in high-risk patients with TIA or minor ischemic stroke as compared with DAPT.

\section{Study Participants}

In total, 5,500 patients will be recruited through 100 emergency departments in general hospitals in China. Two subtypes of patients will be enrolled: (i) acute non-disabling ischemic stroke; and (ii) acute TIA. Ischemic stroke is defined as an abrupt onset of a neurologic deficit that is attributable to acute occlusion of an intracranial vessel. The standard definition of TIA requires that all neurologic signs and symptoms resolve within $24 \mathrm{~h}$, regardless of whether there is imaging evidence of new permanent brain injury; stroke has occurred if the neurologic signs and symptoms last for $>24 \mathrm{~h}$, with image evidence of a new brain infarction. Informed consent will be supported by a patient (or next of kin) information leaflet in Chinese. A consultation meeting will be developed by a study physician to ensure patients and their families understand the study procedure and consent to participation in the trial [14].

The study has been approved by the Medical Ethical Reviewing Committee of the Fourth Military Medical University Medical Center, Xi'an, China. This clinical trial will be conducted in accordance with the principles laid down by the 18th World Medical Assembly (Helsinki, 1964) and all applicable amendments laid down by the World Medical Assemblies and the International Conference on Harmonization guidelines for Good Clinical Practice. 


\section{Study Population and Procedures}

Table 1 shows the inclusion and exclusion criteria of the ADANCE trial.

Figure 1 shows the trial procedure. Participants with suspected TIA or minor stroke will first receive a head computed tomography (CT) to exclude the intracranial hemorrhage and optional head magnetic resonance imaging (MRI) + diffusion weighted imaging (DWI) to confirm the area of infarction. A certified, trained, licensed physician investigator will be required to confirm the diagnosis of TIA or minor ischemic stroke and to calculate the $\mathrm{ABCD}^{2}$ (age, blood pressure, clinical features, duration of TIA, and presence of diabetes) score for subjects with TIA or the NIHSS score for subjects with minor stroke. Once a standardized, structured interview is performed, the data will be recorded in the case report form for each patient. All clinical data, biological samples, and radiological images will be sent to the central study site where a cerebrovascular neurologist will review the data. Demographic, medical, social, and behavioral variables will be determined along with baseline medications. Anthropometry will be conducted with standardized equipment calibrated on a daily basis.

Patients meeting these criteria and offering informed consent will be randomized, and the first dose of study medication will be given within $24 \mathrm{~h}$ of symptom onset.

Patients will be randomized into two groups:

1. Receiving a $75 \mathrm{mg}$ dose of clopidogrel and $75 \mathrm{mg}$ dose of aspirin from day 1 to day 21, with placebo apixaban twice daily.

2. Receiving apixaban $5 \mathrm{mg}$ twice daily, with placebo clopidogrel and placebo aspirin from day 1 to day 21 .

A reduced dose of apixaban ( $2.5 \mathrm{mg}$ twice daily) will be administered if patients have two or more of the following three factors: advanced age $\geq 80$ years, elevated serum creatinine $\geq 1.5 \mathrm{mg} / \mathrm{dL}$, and low body weight $\leq 60 \mathrm{~kg}$.

From day 22 to day 90, all patients will receive a $75 \mathrm{mg}$ dose of clopidogrel long-term antiplatelet therapy. Study visits will be performed at the day of randomization, and at days 7, 22, and 90. From randomization to follow-up visits, the following information will be collected: a neurologic evaluation [modified Rankin Scale (mRS) and NIHSS]; a

Table 1 Inclusion and exclusion criteria

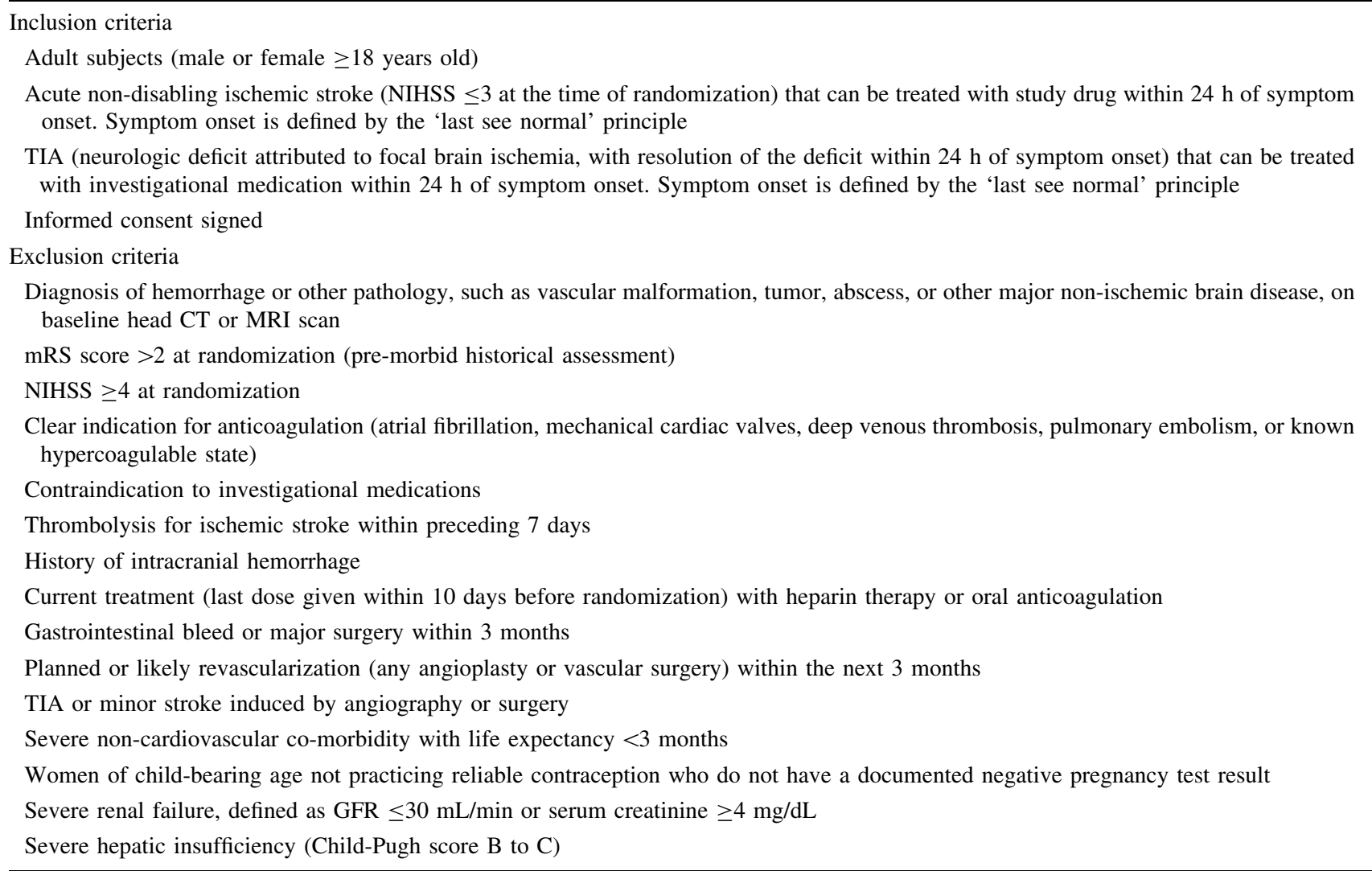

$C T$ computed tomography, GFR glomerular filtration rate, MRI magnetic resonance imaging, $m R S$ modified Rankin Scale, NIHSS National Institutes of Health Stroke Scale, TIA transient ischemic attack 
Fig. 1 Flowchart outlining the trial protocol. bid twice daily, $q d$ once daily, TIA transient ischemic attack

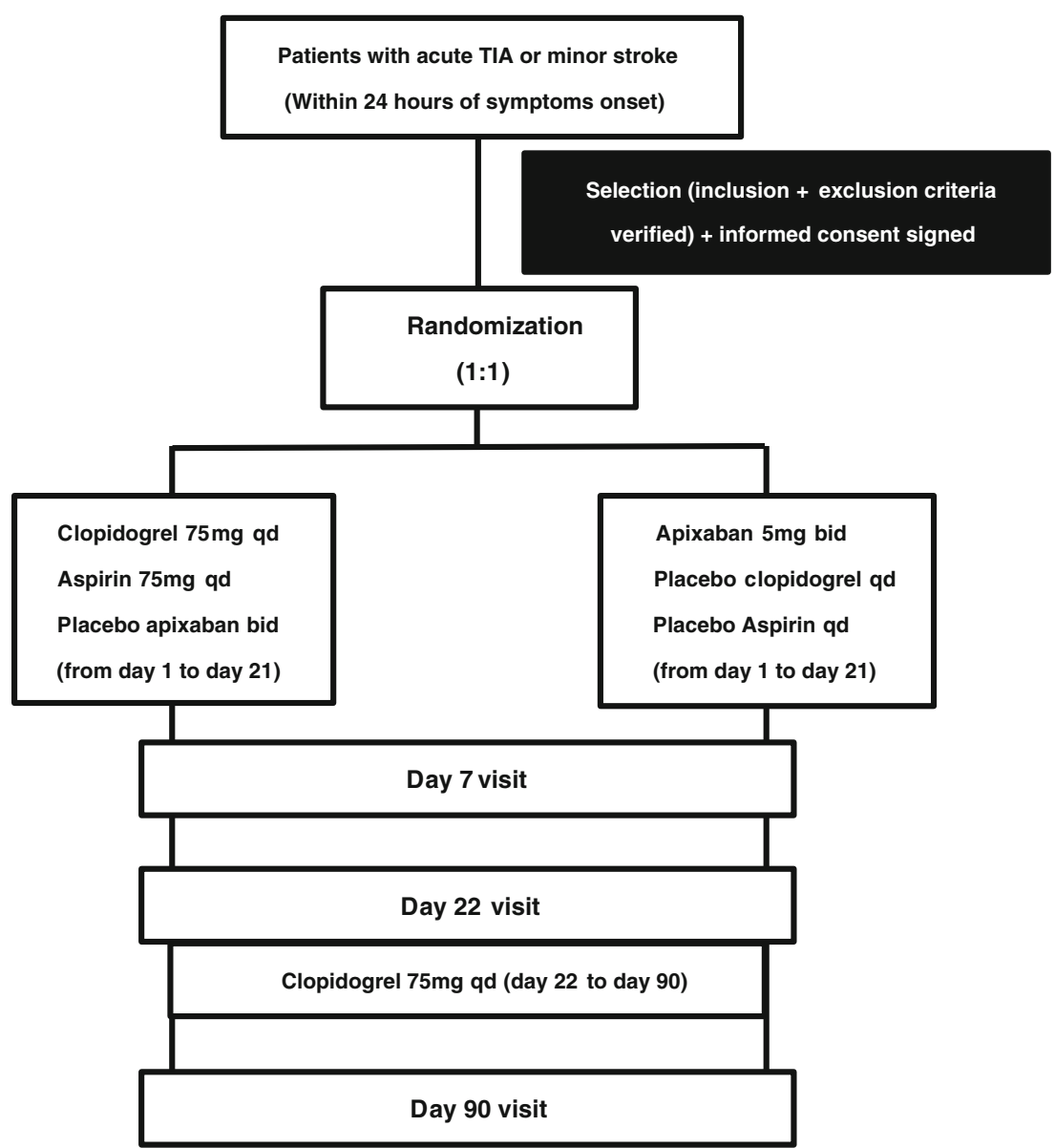

physical examination, including measurement of weight (in $\mathrm{kg}$ ) and vital signs (supine systolic and diastolic blood pressure, heart rate); a neurological dysfunctions assessment; concomitant medications; and adverse events. For ensuring the safety of patients during the study, the trial should be stopped in the following conditions: (i) the rate of intracranial hemorrhage exceeds $5 \%$ or the rate of other hemorrhagic events is more than $10 \%$; or (ii) the rate of 90-day stroke recurrence (ischemic or hemorrhagic) in the DAPT group is $20 \%$ less than in the apixaban group.

An individual not associated with the trial will use a computerized random number generator to randomly assign subjects to group. Allocation concealment will be ensured by using an opaque, sealed envelope containing the group assignment.

\section{Study Outcomes}

The primary efficacy endpoint is the percentage of patients with any new stroke (ischemic or hemorrhage), including fatal stroke, at day 21.

Secondary efficacy endpoints include the following:
1. The percentage of patients at day 90 with new clinical vascular events [ischemic stroke/hemorrhagic stroke/ TIA/myocardial infarction (MI)/vascular death] as a cluster and evaluated individually;

2. mRS score changes given as a percentage with a score of 0-2 versus 3-6 at day 90;

3. Changes in NIHSS scores at day 90; and

4. Efficacy endpoints will also be analyzed stratified by etiologic subtypes (non-intracranial artery diseases vs. intracranial artery diseases), time randomization $(<12$ vs. $\geq 12 \mathrm{~h}$ ), qualifying event (TIA vs. minor stroke), and age (dichotomized at a 5-year cut-point closest to median age).

Safety endpoints include the following:

1. Moderate to severe bleeding events at day 90, according to the GUSTO (Global Utilization of Streptokinase and T-Pa For Occluded Coronary Arteries) definition, including fatal bleeding and symptomatic intracranial hemorrhage, and incidence of symptomatic and asymptomatic intracranial hemorrhagic events at day 90;

2. Total mortality (for any reason) at day 90; and 
3. Adverse events/severe adverse events reported by the investigators.

\section{Randomization, Allocation Concealment, and Blinding}

Subject numbers are to be assigned sequentially as each patient enters the study. After informed consent is received, patients fulfilling the inclusion criteria will be randomized by computer-generated random numbers (Microsoft Excel $^{\circledR}$ 2010, Microsoft Corporation, Redmond, WA, USA) at the statistics research office of the Fourth Military Medical University. A clinical research associate will make opaque blinded envelopes (with consecutive numbers) and deliver them to each participating center. Random allocation is to be performed within $24 \mathrm{~h}$ after a patient is enrolled. The block size and treatment-assignment table would not be available to the researchers until the end of the trial.

The study medication would be stored under the conditions specified on the label in a locked, safe area of the pharmacy department to prevent unauthorized access. Both the study medication and the placebo will be indistinguishable; they will be manufactured by the same company with similar appearances, organoleptic characteristics, and presentations.

In the event of an emergency, an investigator and a clinical pharmacist would decide whether it is necessary to unblind the subject's treatment assignment with the unblinding envelopes provided to the hospital. If unblinding were necessary, the investigator must record the reason, as well as the date and time of the event. In each participating center, an independent researcher would be assigned to collect the trial paper and electronic records. In the central trial coordinating office, an independent research assistant would have access to all unblinded data and maintain confidentiality of patient records.

\section{Statistical Considerations}

The primary null hypothesis of this trial is as follows: in patients with TIA or minor ischemic stroke treated with clopidogrel and aspirin, there is no difference in 90-day risk of stroke (ischemic or hemorrhagic) in those treated with a 21-day regimen of apixaban $5 \mathrm{mg}$ twice daily, if therapy is initiated within $24 \mathrm{~h}$ of symptom onset.

The minimum necessary sample size in the trial is established by the requirement to detect the smallest expected clinically meaningful treatment difference comparing the treatment with placebo. According to previous clinical studies $[4,15,16]$, the 90-day risk of stroke recurrence in the placebo (aspirin) group is about $14 \%$ among high-risk TIA ( $\mathrm{ABCD}^{2}$ score $\geq 4$ ) or minor stroke patients treated with aspirin within $24 \mathrm{~h}$ of symptom onset. The CHANCE study [6] further indicated that, as compared with aspirin monotherapy, clopidogrel and aspirin treatment reduced the risk of recurrent stroke by $32 \%$ and was not associated with increased hemorrhage events. The results from our preliminary study were used to calculate the sample size (unpublished data). The ratios of the 90day stroke recurrence in the DAPT group and apixaban group were 10.47 and $7.96 \%$, respectively. Therefore, we presume that the 90-day risk of stroke recurrence is $10.5 \%$ among high-risk TIA or minor stroke patients with clopidogrel-aspirin therapy within $24 \mathrm{~h}$ of symptom onset. A relative risk reduction of $24 \%$ (relative risk with addition of apixaban is 0.76) is the smallest difference we will attempt to detect. The following equation was used for calculating the sample size:

$n=2 \lambda /\left(2 \sin ^{-1} \sqrt{p 1}-2 \sin ^{-1} \sqrt{p 2}\right)^{2}$

With a two-sided $5 \%$ significance level $(\alpha)$ and an $80 \%$ power $(1-\beta), p 1$ of $0.105, p 2$ of 0.08 , and $\lambda_{0.05,2}$ of 9.63 , the minimal sample size per group was estimated to be 2,600 patients. About 5,500 participants in total in the two groups is the final estimated sample size with $5 \%$ withdrawals (medication non-adherence). Missing values will remain missing, and patients will be censored at their last follow-up assessment (time of clinical event, end of study, or last visit before loss to follow-up).

All analyses will be intent to treat. In these analyses, we will compare apixaban versus DAPT treatment. KaplanMeier estimates of the cumulative risk of a stroke (ischemic or hemorrhagic) event during a maximum of 90 days' follow-up, with hazard ratios and $95 \%$ confidence intervals calculated using a Cox proportional-hazards model and the log-rank test to evaluate the treatment effect will be reported. The Chi-square test of association will be used to compare groups at baseline as appropriate. Logistic regression is to be used to determine the significance of the results obtained. All statistics would be two-sided with $p<0.05$ considered significant.

\section{Study Organization}

The progress of the study would be monitored by the Data and Safety Monitoring Board (DSMB) to ensure the high standards of ethics and patient safety. The DSMB would monitor the trial via scheduled and unscheduled reviews, supervise stopping rules and unmasking, and maintain the 
confidentiality of internal discussions and validity of the reports.

An Executive Committee meeting would be organized to make major decisions. Quality control inspectors are responsible for inspecting the quality of research sites and study data periodically, supervising any quality problem during the trial, and reporting to the Executive Committee. The members of the Steering Committee, including two project directors, will be members of the Executive Committee and will convene monthly (teleconferences or physical meetings) to review the status of the trial and available blinded data; they will take appropriate action regarding the conduct of the study.

An Adjudication Committee charter including membership, role, and responsibilities is to be approved before the start of the trial by the Adjudication Committee and the Executive Committee. This committee will be composed of Academic Members, including an independent statistician, who are not otherwise participating in the trial.

\section{Discussion}

Acute non-disabling cerebrovascular events are common and often portend a disabling stroke. Recently, a few effective therapies have been established according to clinical trials. Aspirin is the mainstay antiplatelet therapy that has been used in patients with an acute cerebrovascular events [17] for a long time. Clopidogrel, a platelet adenosine diphosphate receptor antagonist, has been shown to be as effective for secondary stroke prevention as aspirin, with a similar rate of ischemic stroke, MI, or vascular death [18]. Clopidogrel could not produce maximal inhibition of platelet aggregation in a conventional dose (75 mg/day) for 5 days [19]. This delay presents a problem for an early treatment effect in the management of patients with acute ischemic stroke. Recently, DAPT indicated a reduced risk of recurrent stroke and an acceptable risk of hemorrhage events for high-risk patients with non-disabling cerebrovascular events [6]. Therefore, we can't deny the possibility that there may have been some other medical regimen that could improve outcomes in those patients.

Patients administered with traditional anticoagulants immediately after acute ischemic stroke onset have been reported to have a lower risk of recurrent stroke and an increased rate of bleeding complications, as compared with untreated patients or patients who received an antiplatelet agent [20, 21]. However, in recent times, novel oral anticoagulants, including direct factor Xa inhibitors and direct thrombin inhibitors, have shown advantage in reducing the risk of stroke in patients with $\mathrm{AF}[11,22]$ and preventing or treating venous thromboembolism [10] with fewer hemorrhagic adverse effects and a preferable response compared with traditional anticoagulants [9]. Recently, apixaban (a direct factor Xa inhibitor) has received broad attention for its reliable anticoagulant effect and lower rates of major hemorrhage [23]. Compared with warfarin, apixaban shows better tolerability and a lower rate of systemic embolism, bleeding events, and all-cause mortality [12]. Compared with aspirin, apixaban has been found to have a similar risk of major bleeding and reduced risk of stroke onset in patients with AF [13]. However, the effects of apixaban on the early recurrent stroke risk after TIA and acute minor stroke are still uncertain. The ADANCE trial is a randomized, double-blind, multicenter controlled clinical trial in China. We will assess the effect of a 3-month regimen of apixaban treatment for the first 21 days followed by clopidogrel monotherapy versus a 3-month regimen of DAPT for the first 21 days followed by clopidogrel alone, on reducing the 3-month risk of any stroke when initiated within $24 \mathrm{~h}$ of symptom onset in high-risk patients with TIA or minor stroke.

Acknowledgments Funding for ADANCE was provided by Key Scientific and Technological Project of Shaanxi province. This work was approved by Academic Board and Discipline Construction Committee. The clinical trial was supported by 2013 Key Scientific and Technological Project.

Competing interests The authors declare that they have no competing interests.

Author contributions ZG: the principal investigator for this project who led the conceptualization, design, and funding applications, and made strategic decisions for this research protocol. YF and LH: co-led the conceptualization, design, development, and implementation of this research protocol and contributed to the writing of this manuscript. JWR: led the development of the data management protocol and statistical analysis plan. HJL and JW: contributed to the design, development, and implementation of this research protocol. All authors read and approved the manuscript.

Trial status The trial has not yet begun to enroll patients. The trial is expected to enroll patients in January 2015.

Open Access This article is distributed under the terms of the Creative Commons Attribution Noncommercial License which permits any noncommercial use, distribution, and reproduction in any medium, provided the original author(s) and the source are credited.

\section{References}

1. Mackay J, Mensah G. The atlas of heart disease and stroke. Geneva: World Health Organization; 2004. p. 112.

2. Rothwell PM, Buchan A, Johnston SC. Recent advances in management of transient ischaemic attacks and minor ischaemic strokes. Lancet Neurol. 2006;5(4):323-31.

3. Johnston SC, Gress DR, Browner WS, Sidney S. Short-term prognosis after emergency department diagnosis of TIA. JAMA. 2000;284(22):2901-6. 
4. Coull AJ, Lovett JK, Rothwell PM; Oxford Vascular Study. Population based study of early risk of stroke after transient ischaemic attack or minor stroke: implications for public education and organisation of services. BMJ. 2004;328(7435):326.

5. Hacke W, Bluhmki E, Brozman M, Dávalos A, Guidetti D, Larrue V, et al.; ECASS Investigators. Thrombolysis with alteplase 3 to 4.5 hours after acute ischemic stroke. N Engl J Med. 2008;359(13):1317-29.

6. Wang Y, Zhao X, Liu L, Wang D, Wang C, Wang C, et al.; CHANCE Investigators. Clopidogrel with aspirin in acute minor stroke or transient ischemic attack. N Engl J Med. 2013;369(1): 11-9.

7. Camerlingo M, Salvi P, Belloni G, Gamba T, Cesana BM, Mamoli A. Intravenous heparin started within the first 3 hours after onset of symptoms as a treatment for acute nonlacunar hemispheric cerebral infarctions. Stroke. 2005;36(11):2415-20.

8. Berge E, Abdelnoor M, Nakstad PH, Sandset PM. Low molecular-weight heparin versus aspirin in patients with acute ischaemic stroke and atrial fibrillation: a double-blind randomised study. HAEST Study Group. Heparin in acute embolic stroke trial. Lancet. 2000;355(9211):1205-10.

9. Furie KL, Goldstein LB, Albers GW, Khatri P, Neyens R, Turakhia MP, et al.; American Heart Association Stroke Council; Council on Quality of Care and Outcomes Research; Council on Cardiovascular Nursing; Council on Clinical Cardiology; Council on Peripheral Vascular Disease. Oral antithrombotic agents for the prevention of stroke in nonvalvular atrial fibrillation: a science advisory for healthcare professionals from the American Heart Association/American Stroke Association. Stroke. 2012 Dec;43(12):3442-53.

10. Agency EM. Eliquis ${ }^{\circledR}$ - summary of product characteristics. 2011. http://www.ema.europa.eu/docs/en_GB/document_library/ EPAR_-_Product_Information/human/002148/WC500107728.pdf. Accessed 18 Aug 2014.

11. FDA. FDA approves Eliquis to reduce the risk of stroke, blood clots in patients with non-valvular atrial fibrillation. 2012. http:// www.fda.gov/NewsEvents/Newsroom/PressAnnouncements/ucm 333634.htm. Accessed 18 Aug 2014.

12. Granger CB, Alexander JH, McMurray JJ, Lopes RD, Hylek EM, Hanna M, et al.; ARISTOTLE Committees and Investigators. Apixaban versus warfarin in patients with atrial fibrillation. N Engl J Med. 2011;15;365(11):981-92.

13. Flaker GC, Eikelboom JW, Shestakovska O, et al. Bleeding during treatment with aspirin versus apixaban in patients with atrial fibrillation unsuitable for warfarin: the apixaban versus acetylsalicylic acid to prevent stroke in atrial fibrillation patients who have failed or are unsuitable for vitamin $\mathrm{K}$ antagonist treatment (AVERROES) trial. Stroke. 2012;43(12):3291-7.

14. Allmark P, Mason S. Improving the quality of consent to randomised controlled trials by using continuous consent and clinician training in the consent process. J Med Ethics. 2006;32(8): 439-43.

15. Johnston SC, Rothwell PM, Nguyen-Huynh MN, et al. Validation and refinement of scores to predict very early stroke risk after transient ischaemic attack. Lancet. 2007;369(9558):283-92.

16. Kennedy J, Hill MD, Ryckborst KJ, et al. Fast assessment of stroke and transient ischaemic attack to prevent early recurrence (FASTER): a randomised controlled pilot trial. Lancet Neurol. 2007;6(11):961-9.

17. Jauch EC, Saver JL, Adams HP Jr, et al. Guidelines for the early management of patients with acute ischemic stroke: a guideline for healthcare professionals from the American Heart Association/American Stroke Association. Stroke. 2013;44(3):870-947.

18. CAPRIE Steering Committee. A randomised, blinded, trial of clopidogrel versus aspirin in patients at risk of ischaemic events (CAPRIE). CAPRIE Steering Committee. Lancet. 1996;348 (9038):1329-39.

19. Qureshi AI, Luft AR, Sharma M, et al. Prevention and treatment of thromboembolic and ischemic complications associated with endovascular procedures: Part II-clinical aspects and recommendations. Neurosurgery. 2000;46(6):1360-75.

20. International Stroke Trial Collaborative Group. The International Stroke Trial (IST): a randomised trial of aspirin, subcutaneous heparin, both, or neither among 19435 patients with acute ischaemic stroke. International Stroke Trial Collaborative Group. Lancet. 1997;349(9065):1569-81.

21. Low molecular weight heparinoid, ORG 10172 (danaparoid), and outcome after acute ischemic stroke: a randomized controlled trial. The Publications Committee for the Trial of ORG 10172 in Acute Stroke Treatment (TOAST) Investigators. JAMA. 1998;279(16):1265-72.

22. Connolly SJ, Ezekowitz MD, Yusuf S, et al. Dabigatran versus warfarin in patients with atrial fibrillation. $\mathrm{N}$ Engl $\mathrm{J}$ Med. 2009;361(12):1139-51.

23. Gong IY, Kim RB. Importance of pharmacokinetic profile and variability as determinants of dose and response to dabigatran, rivaroxaban, and apixaban. Can J Cardiol. 2013;29(7 Suppl): S24-33. 